\title{
Determination of the earth's surface deformations in the "Kiyzassky" open pit according to Sentinel-1 Data
}

\author{
Leonid Mikov*, Semion Popov \\ Federal Research Center for Information and Computational Technologies, Geoinformation \\ Modelling Lab, 630090 Novosibirsk, Russia
}

\begin{abstract}
The paper deals with the issues of assessment of the condition and changes in the land surface on the territory of the Kiizassky open pit (Kemerovo region) because of the landslide that occurred in June 2019. The application of the multi-pass series of Sentinel-1 satellite radar data using the Small Baseline Subset (SBaS) method to determine the Earth surface displacement dynamics using constructed vertical displacement maps is demonstrated..
\end{abstract}

\section{Introduction}

Currently Kuzbass is one of the main mining regions in Russia. High technogenic load associated with the annual increase in coal production leads to the redistribution of stressstrain state of significant volumes of rock mass with adverse consequences for the environment [1]. Deformations of the Earth's surface in such regions, resulting from uneven subsidence and horizontal movements of rocks, reach large territories and are the source of emergency situations, which, in turn, may harm not only the environment and the operating mode of the enterprise, but also human lives. For example, in June 2019, a landslide occurred at one of the Kiyzassky open-pit mine. As a result, the supports of nine power transmission lines were damaged and the bed of the Bolshoi Kiyzas River was blocked by 750 meters in length and about five meters in height [2] (Fig. 1) [3].

\section{InSAR data processing methods}

Today, satellite radar data are actively used to determine the deformations of the earth's surface. Their advantage is the relatively low price per square kilometer, as well as the independence of the shooting from weather conditions and time of day. To process such data, 3 main methods have been developed:

- differential interferometry (DinSAR). Used to process two images

\footnotetext{
*Corresponding author: mikov@,ict.sbras.ru
} 
- method of persistent scatterer (PS). It is used for point estimation of deformations based on a multi-pass series of images
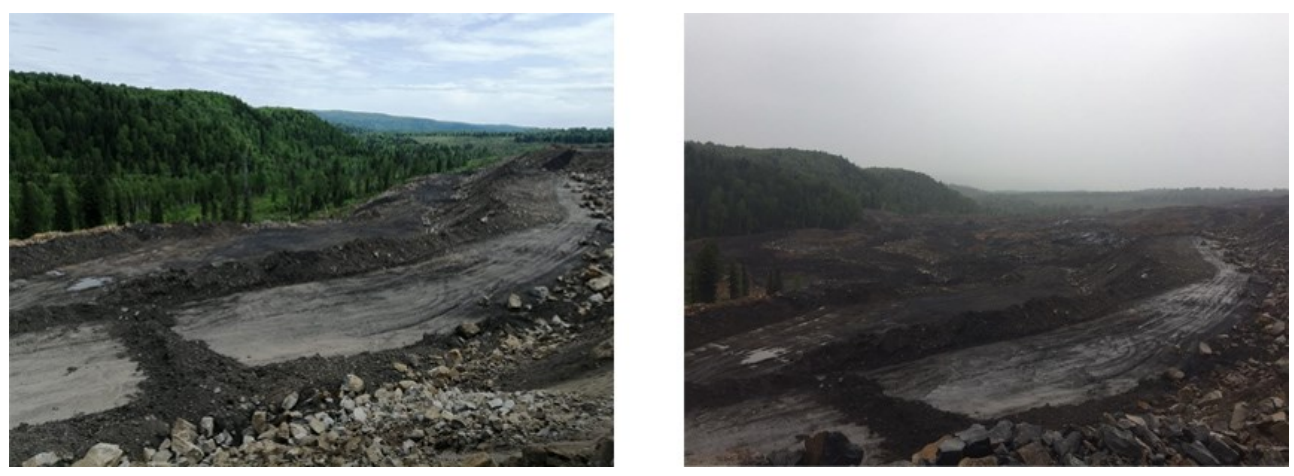

Fig. 1. Photo of the Kiyzassky open pit stockpile before the landslide (left) and after the landslide (right)

- small baseline subset method (SBaS). Used for areal strain assessment based on a multipass series of images.

The principle of radar interferometry is as follows. Each pixel of the satellite image contains information about the real and imaginary part of the wave reflected from the part of the Earth's surface and received by the satellite. Thus, the satellite image has two components: real and imaginary part, which can be converted into the amplitude (brightness) and phase components of the image. Note that a typical size of the Earth surface area in one pixel of the image exceeds a square meter, which makes it impossible to determine millimeter offsets by the brightness component of the image. For this purpose, the analysis of phase components of the image set is used.

Based on a set of phase components of SAR images received by the satellite at different points in time (with periodicity, for example, one image per day), the change in phase component is calculated over time. The phase component change is due to a linear dependence with the difference in the course of the wave reflected from the surface, and thus it is possible to measure the vertical displacements of objects on the Earth's surface, comparable to the signal length of the satellite, which has the order of a few centimeters. [4]

The main difference between the methods is the number of source data to be processed. For example, for the method of differential interferometry a pair of radar images taken with a small time interval (from several days to a couple of weeks) is used. As a result, we get a map of the Earth's surface displacements that occurred between shots. The SBaS method requires at least three images, while the PS method requires at least thirty. The last two methods allow us to estimate the dynamics of the Earth surface changes over a long period of time (from several months to several years). Let us consider all these methods in detail.

Differential interferometry is a classical pairwise method for calculating area displacements. It includes the following stages: interferogram formation, filtering and coherence calculation, phase unwrapping, orbital correction and refinement, phase to displacement recalculation. At the output, we get a map of the Earth's surface displacements.

PS (Persistent Scatterers) method - calculation of displacements of point targets which are permanent reflectors for radar satellite. It allows to measure detailed displacements on infrastructure objects. Used, as a rule, for areas with urban terrain. Includes the same stages 
as the SBaS method. At the output we also get a map of the average velocities of changes in surface displacements.

SBaS method (Small Baseline subset) - calculates accurate area displacements using a series of space images with small baselines. It includes the following stages: interferogram formation with further filtration and unwrapping, correction of orbital errors, first inversion, second inversion (atmospheric correction) and orthorrectification, geocoding of processing results. At the output we get a dot vector file, which is a map of average velocities of Earth surface displacements change. [4-5]

\section{Results and disscution}

In our work, we used the latter method since it allows us to reveal the dynamics of areal deformations of the earth's surface over a long period of time.

The object of the study was the northern part of the stockpile of the Kiyzassky open pit mine, on the territory of which a landslide occurred. For calculations, 11 radar images were selected from the Sentinel-1B spacecraft for the period from 04/12/2019 to 07/29/2019 with a shooting frequency of 12 days. This spacecraft performs imaging in C-band with $5 \times 20$ meters/pixel resolution (in Interferometric Wide Swath mode) and with VV and VH polarization.

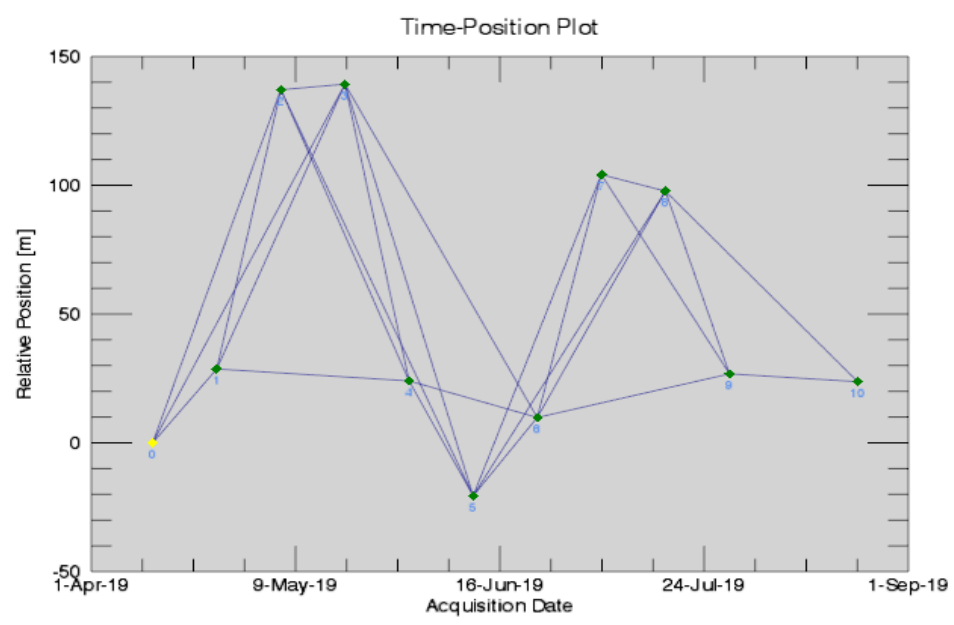

Fig. 2. Time-Position plot

Usually, the original radar image covers a large territory (several tens of thousands of square kilometers), so to reduce the time spent on processing a separate area of interest of about five square kilometers to the northeastern part of the "Kiizassky" section was allocated. To reduce the amount of time and intermediate processing data, VV polarization imagery was used. In addition, it has been experimentally determined that such polarization is better suited for determining displacements than $\mathrm{VH}$. The whole processing was performed in the SARscape software package. 
At the first stage, combinations of image pairs (a master image - an auxiliary image) and a network of connections (Fig. 2) were determined, which are used to form several differential interferograms.

It should be noted that those image pairs, which do not exceed the baseline threshold value (the default value is $500 \%$ ), are suitable for processing. If the value is exceeded, pairs are excluded from further processing.

Then standard interferometric processing was performed with coregistration, filtering and phase unwrapping of image pairs selected at the first stage. Multilooking parameters equal to 1 in azimuth and 5 in range were used for image co-registration. To improve accuracy, we used the SRTM v4 digital elevation model with a spatial resolution of about 30 meters ( 1 arc second). Noise filtration was performed with the help of Goldstein filter. After that, the procedure of phase progression correction from the Earth ellipsoid was performed [6], where the topographic phase was removed from the obtained interferograms. The next step was two inversion stages. At the first stage, the resulting height and displacement rate are determined. The second step is to obtain offsets by dates, which are filtered to remove atmospheric phase components. Finally, geocoding of the results is performed with filtration by velocity and altitude to remove areas with high noise reading (areas with rich vegetation).

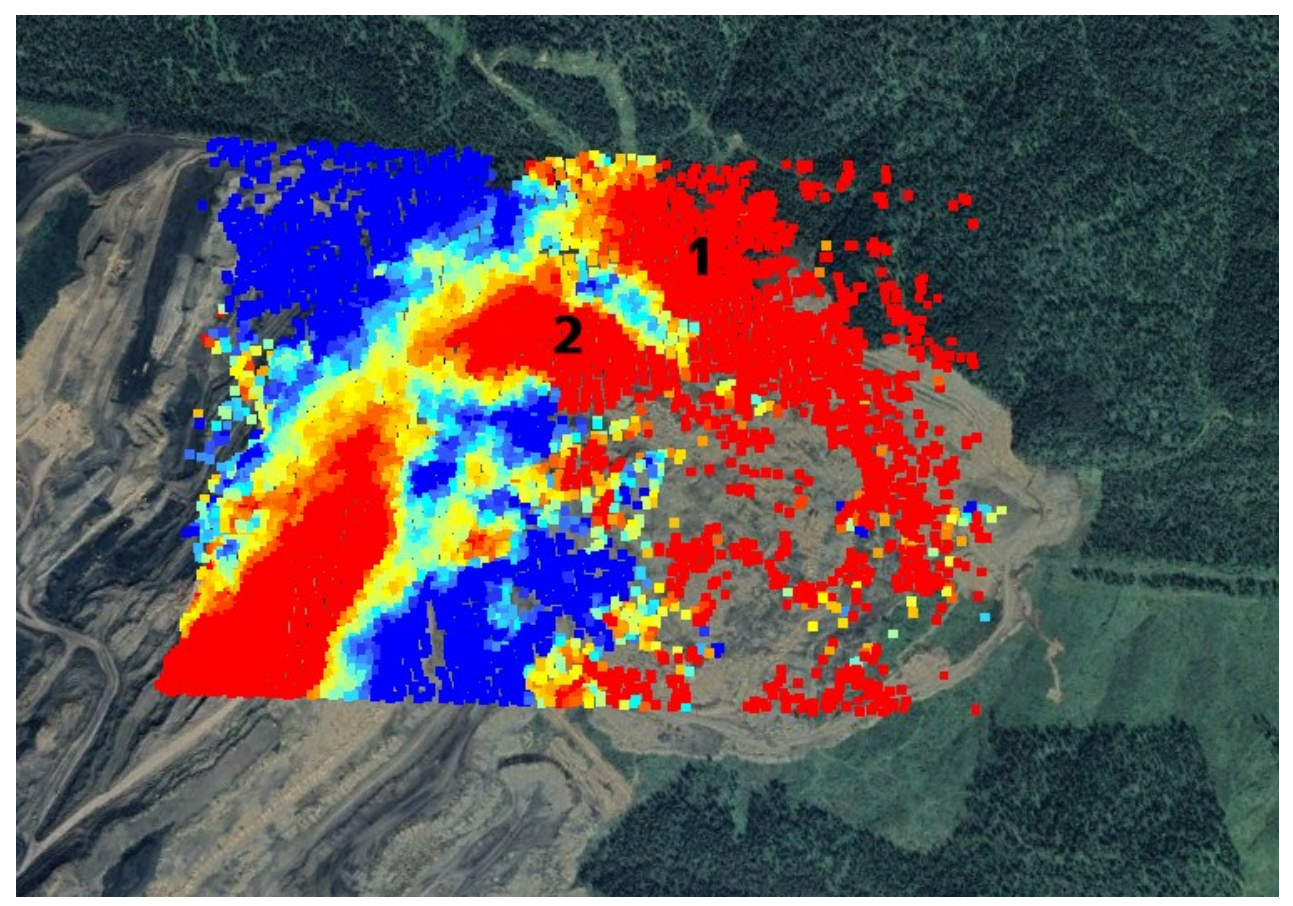

Fig. 3. Map of average velocities of vertical displacements.

During the processing of the initial images by the SBaS method, a map of the average rates of change in the vertical displacements of the earth's surface was built (Fig. 3). This map is a vector file with 4909 points. Each point contains a set of attributes, such as geographic coordinates, average displacement rate value, etc. Points with negative vertical displacements are highlighted in blue, which indicate subsidence of the earth's surface, and in red, elevations of the earth's surface. 
On the map, figure 2 indicates the stockpile of the Kiizassky section, figure 1 - a section filled with rocks formed after the landslide. As can be seen from Figure 2, a dip is detected between sections 1 and 2, which means that rocks are coming off the side of the stockpile. In section 1, positive vertical displacements associated with an increase in the surface height due to the descent of rocks from the side and their further collision with the hillside are detected (Fig. 1).

Separately, it should be noted that the average displacement rates for the period from May 30, 2019 to June 11, 2019 (the landslide occurred on the night of June 7) increased threefold. The values of the average displacement rates over the observation period ranged from $-170 \mathrm{~mm} /$ year to $+200 \mathrm{~mm} /$ year.

Thus, the use of radar data from Sentinel-1 spacecraft is expedient for landslide monitoring, especially in areas with intensive mining operations. The obtained results may become the basis for the development of monitoring systems and landslide prevention activities.

The reported study was funded by RFBR and Kemerovo region, project number 20-47-420002.

\section{References}

1. D. Raucoules, C. Colesanti, C. Carnec Use of SAR interferometry for detecting and assessing ground subsidence. Comptes Rendus Geosci, 339, 289-302, (2007)

2. Stali izvestny prichiny CHP na razreze «Kijzasskij» v Kuzbasse https://gazeta.a42.ru/lenta/news/57608_stali-izvestny-prichiny-chp-na-razrezekiizasskii-v-kuzbasse. Cited January 20, 2020.

3. Opolzen' na razreze Kijzasskij snyos shest' opor LEP, zavalil les i reku Kijzas. http://kuzpress.ru/incident/09-06-2019/68082.html. Cited January 20, 2020.

4. Kantemirov YU.I. Kratkiye teoreticheskiye osnovy radarnoy interferometrii yeye mnogoprokhodnykh variatsiy PS i SBaS // Geomatika. -2012.-№1.-S. 22-26

5. Rosen P A, Hensley S, Joughin I R, Li F K, Madsen SN, Rodriguez E, Goldstein RM Synthetic aperture radar interferometry. Proc IEEE 88(3), 333-382, (2000)

6. Sputnikovyye tekhnologii v geofizike. http://media.ls.urfu.ru/566/1545/3670 Cited October 10, 2020. 